Surveys in Differential Geometry XV

\title{
Anomaly Constraints and String/F-theory Geometry in 6D Quantum Gravity
}

\author{
Washington Taylor
}

\begin{abstract}
Quantum anomalies, determined by the Atiyah-Singer index theorem, place strong constraints on the space of quantum gravity theories in six dimensions with minimal supersymmetry. The conjecture of "string universality" states that all such theories which do not have anomalies or other quantum inconsistencies are realized in string theory. This paper describes this conjecture and recent work by Kumar, Morrison, and the author towards developing a global picture of the space of consistent $6 \mathrm{D}$ supergravities and their realization in string theory via F-theory constructions. We focus on the discrete data for each model associated with the gauge symmetry group and the representation of this group on matter fields. The $6 \mathrm{D}$ anomaly structure determines an integral lattice for each gravity theory, which is related to the geometry of an elliptically fibered Calabi-Yau three-fold in an F-theory construction. Possible exceptions to the string universality conjecture suggest novel constraints on low-energy gravity theories which may be identified from the structure of F-theory geometry.
\end{abstract}

\section{Introduction}

String theory is not yet a mathematically well-defined system. Nonetheless, the components of this theory which have been pieced together so far suggest a framework in which quantum physics and general relativity are unified. In certain limits it seems that the theory can be described geometrically and gives rise to 4D "vacuum solutions", in which space-time has four macroscopic dimensions and additional microscopic dimensions are curled

The author would like to thank collaborators Vijay Kumar and David Morrison, with whom the work described in this paper was carried out. The author is immensely grateful to IMS for regular stimulating discussions and interactions over the last two decades, and for his optimism and constant support over the years. This research was supported by the DOE under contract \#DE-FC02-94ER40818.

(C)2011 International Press 
up into a compact manifold. Each $4 \mathrm{D}$ string vacuum solution gives rise to a low-energy physical theory in four dimensions which generally is a theory of gravity coupled to Yang-Mills theory with matter fields transforming under the Yang-Mills symmetry group. The vast landscape of possible fourdimensional string theory vacua presents a major challenge for physicists who hope to derive predictions for observable physics from string theory. String theorists have struggled with this problem since the early days of the subject, when it was realized that ten-dimensional string theory could be compactified to four dimensions on any Calabi-Yau threefold, giving a wide variety of low-energy theories. Efforts to remove unphysical massless scalar fields associated with Calabi-Yau moduli, such as through inclusion of generalized magnetic fluxes, have sharpened the problem further by generating exponentially large (or infinite) classes of vacua associated with each Calabi-Yau geometry (for a review of recent work in this direction see e.g. [1]). Beyond the enormous range of string vacua which can be constructed using this and other known mechanisms, it may be that even larger classes of string vacua can be constructed using less well-understood non-geometric compactifications. Despite the wide range of possible string vacuum constructions, the range of low-energy theories which exhibit no known quantum inconsistencies when coupled to gravity seems to be far vaster still. The term "swampland" was coined by Vafa to characterize the set of low-energy gravity theories with no known inconsistencies which cannot be realized in string theory [2]. At present, the swampland for four dimensions appears to be very large, and we lack tools powerful enough to make strong global statements about the space of consistent quantum gravity theories.

In six space-time dimensions, however, a global understanding of the space of supersymmetric string vacua may be within reach. In $4 k+2$ dimensions, quantum anomalies can lead to a violation of diffeomorphism or YangMills invariance. The condition that such anomalies are absent strongly constrains the range of possible quantum-consistent supergravity theories in six dimensions. The following conjecture was presented at the conference "Perspectives in Mathematics and Physics," in honor of I. M. Singer, and stated more precisely in the paper $[\mathbf{3}]$.

CONJECTURE 1.1 ("String Universality in six dimensions"). All $\mathcal{N}=$ $(1,0)$ supersymmetric theories in $6 D$ with one gravity and one tensor multiplet which are free of anomalies or other quantum inconsistencies admit a string construction.

In the statement of this conjecture, $\mathcal{N}=(1,0)$ supersymmetry is the minimal possible amount of supersymmetry in six dimensions. Supersymmetry is a graded symmetry relating bosonic (commuting) to fermionic (anticommuting) fields. The gravity and tensor multiplets are combinations of fields transforming under irreducible representations of the supersymmetry algebra. We describe some relevant features of these multiplets in more detail in the following section. Over the last year, further work with V. Kumar [4] 
and with V. Kumar and D. Morrison $[\mathbf{5}, \mathbf{6}]$ has led to a global picture of the space of constraints on these low-energy supergravity theories, and an explicit correspondence with the F-theory description of string vacua, not only for theories with one tensor multiplet, but also for a much richer class of theories with multiple tensor multiplets. We have proven that when the number of tensor multiplets is less than 9 , there are only a finite number of distinct gauge groups and matter representations which can appear in consistent low-energy theories $[\mathbf{4}, \mathbf{6}]$. This analysis has also, however, produced some apparent counterexamples to the conjecture. Either these counterexamples represent new classes of string vacua, which cannot be realized using conventional F-theory, or there must be additional stringy constraints on the space of possible low-energy theories. In the latter case, if the conjecture is correct, it may be the case that these constraints can be identified from the low-energy data describing the theory as obstructions to a consistent quantum UV completion. The analogue of Conjecture 1.1 in 10 dimensions was recently proven by Adams, DeWolfe, and the author by demonstrating the quantum inconsistency of the two models with gauge groups $U(1)^{496}$ and $E_{8} \times U(1)^{248}$ which were previously thought to lie in the swampland [7]. Further progress towards proving or disproving the string universality conjecture in 6 dimensions will require achieving a better mathematical or physical understanding of the set of possible string constructions and lowenergy constraints.

In this paper we describe some of the context and motivation for the $6 \mathrm{D}$ string universality conjecture, and give a somewhat self-contained presentation of some of the core mathematical arguments underlying recent progress in understanding the relevant space of $6 \mathrm{D}$ supergravity theories. The grouptheoretic structure of the anomaly cancellation conditions is reviewed in Section 2 and used in Section 3 to place bounds on the set of gauge groups and matter representations which can be realized in $\mathcal{N}=1$ supersymmetric $6 \mathrm{D}$ theories of gravity coupled to Yang-Mills theory. From the anomaly conditions, an integral lattice $\Lambda$ can be associated with each such low-energy supergravity theory. In Section 4, we describe the connection with F-theory, where $\Lambda$ is a sublattice of the lattice of integral second homology classes on a complex surface which is the base of an elliptic fibration. In Section 5 we give some explicit examples of $6 \mathrm{D}$ theories and describe some apparent counterexamples to the conjecture. This leads to a discussion in the concluding section 6 of possible new constraints on low-energy supergravity theories.

\section{6D supergravity: gauge groups, matter representations, and anomalies}

In this section we describe some of the key mathematical structures in $6 \mathrm{D}$ supergravity theories. In particular, we show how the set of possible theories is constrained by using group theory to analyze quantum anomaly constraints. Our focus here is on topological, rather than analytic aspects of 
these theories. Thus, we are primarily interested in classifying the discrete data associated with the set of quantum fields in the theory, in particular the Yang-Mills symmetry group and associated representations. The following abbreviated outline of the structure of $6 \mathrm{D}$ supergravity theories highlights this discrete structure, and elides many of the details of the physics of these theories. For a more detailed description of $6 \mathrm{D} \mathcal{N}=(1,0)$ supergravity theories, see the original papers $[\mathbf{1 0}, \mathbf{1 1}, \mathbf{1 2}]$.

2.1. Gauge groups and matter fields. Any $6 \mathrm{D}$ supergravity theory with $\mathcal{N}=(1,0)$ supersymmetry is characterized by the following discrete data

$$
\begin{array}{cl}
T & T \in \mathbb{Z}, T \geq 0 \quad \text { [number of anti-self-dual tensor fields] } \\
G & \text { compact finite-dimensional Lie group [gauge group] } \\
M & \text { finite-dimensional representation of } G \text { [matter content] }
\end{array}
$$

This data describes the set of dynamical quantum fields in the supergravity theory. As mentioned above, the fields in the theory live in supersymmetry multiplets-that is, in representations of the supersymmetry algebra of the theory. There are four types of supersymmetry multiplets appearing in $\mathcal{N}=(1,0)$ theories. Each theory contains a single "gravity" multiplet. The gravity multiplet includes among its bosonic components a symmetric tensor field $g_{\mu \nu}$, which describes the metric on the $6 \mathrm{D}$ space-time manifold $\mathcal{M}$, and an antisymmetric two-form field $B_{\mu \nu}^{0}$, which satisfies a self-dual condition $\sim d B^{0}={ }^{*} d B^{0} . T$ denotes the number of "tensor" multiplets, which each include an anti-self-dual two-form field $B_{\mu \nu}^{i}$ and a scalar field $\phi^{i}, i=1, \ldots T$. Each model has some some number of "vector" multiplets, which contain one-form gauge fields $A_{\mu}$ describing a connection on a principal $G$-bundle over $\mathcal{M}$. Each model also has a set of "hyper" multiplets, each containing four scalar fields $\varphi^{a}$. These scalar fields parameterize a quaternionic Kähler manifold, and transform under a (generally reducible) representation $M$ of the gauge group $G$. Each multiplet, in addition to the bosonic fields just mentioned, also contains fermionic (Grassmann) fields related to the bosonic fields through supersymmetry. We will not be concerned with many details of the field structure in the analysis here; the principal data we are concerned about are the data $T, G, M$ characterizing the number of tensor multiplets, the gauge group, and the matter representation. Furthermore, we will focus on the nonabelian structure of the gauge group, taking $G$ to be semisimple and ignoring $U(1)$ factors. We write $G$ as a product of simple factors $H_{i}$

$$
G=H_{1} \times H_{2} \times \cdots \times H_{k} .
$$

We denote the number of vector multiplets associated with generators of $G$ by $V=\operatorname{dim} G$, and the number of hypermultiplets transforming (trivially or nontrivially) under $G$ by $H=\operatorname{dim} M$. 
2.2. Anomalies. Not all possible combinations of discrete data $T, G, M$ can be associated with consistent supersymmetric quantum theories of gravity. Quantum anomalies impose strong constraints on the set of possible theories. In fact, the condition that a theory be free of anomalies, along with a simple physical sign constraint on the structure of the gauge fields, is sufficient to prove that when $T<9$ there are only a finite number of possible nonabelian gauge groups $G$ and matter representations $M$ possible for a consistent quantum supergravity theory. This was proven for $T=1$ in [4], and for $T<9$ in [6]. After summarizing the anomaly conditions in this subsection, we present the key arguments in the proof of finiteness in the following subsection.

A quantum anomaly is a breakdown at the quantum level of a classical symmetry. One way to understand anomalies (Fujikawa [13]) is in terms of the path integral formulation of a quantum theory. A quantum theory containing fermion fields $\psi, \bar{\psi}$ can be defined through a path integral

$$
Z=\int \mathcal{D} \psi \mathcal{D} \bar{\psi} e^{i S(\psi, \bar{\psi})},
$$

where the action $S(\psi, \bar{\psi})=\bar{\psi} \not D \psi+\cdots$ contains a Dirac operator $\not D$ and is invariant under local gauge transformations $\psi \rightarrow g \psi$. For chiral fermionic fields, nontrivial transformation of the measure factor $\mathcal{D} \psi \mathcal{D} \bar{\psi}$ leads to a quantum violation of the classical gauge symmetry. In terms of Feynman diagrams, such quantum anomalies appear in one-loop computations, and cannot be removed by any choice of the regularization prescription used to remove infinities from the quantum calculation. For gravitational theories, chiral fermions and self-dual/anti-self-dual antisymmetric tensor fields lead to similar anomalies associated with violations of local diffeomorphism invariance [14].

As was shown in $[\mathbf{1 5}, \mathbf{1 6}]$, gauge and gravitational anomalies are determined by characteristic classes associated with the index of the Dirac operator coupled to the appropriate vector potential. The anomaly in $D$ dimensions is associated with an index in $D+2$ dimensions. Thus, in particular, gauge, gravitational, and mixed gauge-gravitational anomalies in six dimensions are associated with an 8-form $I_{8}$ containing terms built from the geometric and Yang-Mills curvatures, such as $\operatorname{tr} R^{4}, \operatorname{tr} F^{4}, \operatorname{tr} R^{2} \operatorname{tr} F^{2}, \ldots$ It was shown by Green and Schwarz $[\mathbf{1 7}]$ that the anomaly in some 10dimensional supergravity theories can be cancelled by classical (tree-level) terms associated with exchange of quanta associated with antisymmetric two-form fields. This mechanism was extended to six dimensions in [18] for theories with one tensor multiplet, and in $[\mathbf{1 9}]$ for theories with any number of tensor multiplets. Anomalies in six-dimensional supergravity theories with a single supersymmetry can be cancelled by the Green-Schwarz-Sagnotti mechanism if the anomaly polynomial $I_{8}$ can be written in the form

$$
I_{8}(R, F)=\frac{1}{2} \Omega_{\alpha \beta} X_{4}^{\alpha} X_{4}^{\beta}
$$


where $X_{4}^{\alpha}$ is a 4 -form taking the form

$$
X_{4}^{\alpha}=\frac{1}{2} a^{\alpha} \operatorname{tr} R^{2}+\sum_{i} b_{i}^{\alpha}\left(\frac{2}{\lambda_{i}} \operatorname{tr} F_{i}^{2}\right) .
$$

Here, $a^{\alpha}$ and $b_{i}^{\alpha}$ are vectors in the space $\mathbb{R}^{1, T}$ carrying a symmetric bilinear form $\Omega_{\alpha \beta}$. The normalization factor $\lambda_{i}$ is chosen depending on the gauge factor $H_{i}$ such that minimal instantons have compatible normalization factors. For example, $\lambda_{S U(N)}=1$, while $\lambda_{E_{8}}=60$.

The anomaly polynomial does not specify the vectors $a^{\alpha}, b_{i}^{\beta}$, but only constrains the $S O(1, T)$ invariant quantities $a^{2}, a \cdot b_{i}, b_{i} \cdot b_{j}$, where the inner product is taken with respect to the form $\Omega$. A detailed calculation of the anomaly arising from all chiral fields in the theory (see $[\mathbf{1 8}, \mathbf{2 0}, \mathbf{2 1}$ ] for details) gives the form of the complete anomaly polynomial as a function $I(R, F)$ of the gravitational and Yang-Mills curvature two-forms $R$ and $F$.

In order for the anomaly to factorize as in $(2.3), I_{8}$ cannot contain terms proportional to $\operatorname{tr} R^{4}$, which gives the condition

$$
H-V=273-29 T \text {. }
$$

The $\operatorname{tr} F^{4}$ contribution to the total anomaly must also cancel for each gauge group factor, giving the condition

$$
B_{A d j}^{i}=\sum_{R} x_{R}^{i} B_{R}^{i}
$$

where we use the group theory coefficients $A_{R}, B_{R}, C_{R}$ defined through

$$
\begin{aligned}
& \operatorname{tr}_{R} F^{2}=A_{R} \operatorname{tr} F^{2} \\
& \operatorname{tr}_{R} F^{4}=B_{R} \operatorname{tr} F^{4}+C_{R}\left(\operatorname{tr} F^{2}\right)^{2}
\end{aligned}
$$

and where $x_{R}^{i}$ denotes the number of hypermultiplets transforming in irreducible representation $R$ under gauge group factor $H_{i}$.

The remaining conditions that the anomaly factorize relate inner products between the vectors $a, b_{i}$ to group theory coefficients and the representations of matter fields

$$
\begin{aligned}
a \cdot a & =9-T \\
a \cdot b_{i} & =\frac{1}{6} \lambda_{i}\left(A_{a d j}^{i}-\sum_{R} x_{R}^{i} A_{R}^{i}\right) \\
b_{i} \cdot b_{i} & =-\frac{1}{3} \lambda_{i}^{2}\left(C_{a d j}^{i}-\sum_{R} x_{R}^{i} C_{R}^{i}\right) \\
b_{i} \cdot b_{j} & =\lambda_{i} \lambda_{j} \sum_{R S} x_{R S}^{i j} A_{R}^{i} A_{S}^{j}
\end{aligned}
$$

where $x_{R S}^{i j}$ denotes the number of hypermultiplets transforming in irreducible representations $R, S$ under the gauge group factors $H_{i}, H_{j}$. 


\section{Constraints from anomalies}

The conditions (2.5-2.12) place strong constraints on the range of possible theories compatible with anomaly cancellation, particularly for small values of $T$. For one thing, these conditions imply that the inner products $a \cdot a, a \cdot b_{i}, b_{i} \cdot b_{j}$ are all integral and hence that the vectors $a, b_{i} \in \mathbb{R}^{(1, T)}$ span an integral lattice $\Lambda$ (which may be degenerate, for example if several $b_{i}$ are equal). For gauge group factors $H_{i}$ with an irreducible quartic invariant (nonzero $B$ 's), the integrality of the inner products follows from group theory identities which show that, e.g., $3 \mid \lambda_{i}^{2} C_{R}^{i}$ for all representations $R$. For group factors with no irreducible quartic invariant, such as $S U(2), S U(3), E_{8}$, integrality of the inner products depends upon cancellation of additional global anomalies. Details of the proof of integrality are given in [6].

Beyond the integral lattice structure imposed by (2.9-2.12), these conditions also provide strong bounds on the set of possible gauge groups and matter content. To give a finite bound to the set of allowed $G, M$ for small $T$ we need to impose an additional condition, associated with the constraint that each gauge group component has a sensible physical description without instabilities. This condition amounts to the constraint that there exists a vector $j \in \mathbb{R}^{1, T}$ such that

$$
j \cdot b_{i}>0 \forall i, \quad j^{2}=1 .
$$

The vector $j$ in this equation corresponds to the scalar fields in the $T$ tensor multiplets of the supergravity theory.

Given these conditions, we can prove

Theorem 3.1. For $T<9$, there are a finite number of distinct nonabelian gauge groups $G$ and matter representations $M$ satisfying (2.5-2.12), such that (3.1) holds for some $j$.

This theorem is proven in [4] for $T=1$, and in $[6]$ for $T<9$. The proof essentially relies upon geometry in the $(1, T)$ signature space containing the vectors $a, b_{i}, j$. We present here the main arguments in the proof.

Proof. The proof proceeds by contradiction. We assume that there is an infinite family of models with nonabelian gauge groups $\left\{G_{\gamma}\right\}$. For any given model in the family we decompose the gauge group into a product of simple factors $G_{\gamma}=H_{1} \times H_{2} \times \cdots \times H_{k}$. There are a finite number of groups $G$ with dimension below any fixed bound. For each fixed $G$, there are a finite number of representations whose dimension is below the bound (2.5) on the number of hypermultiplets. Thus, any infinite family $\left\{G_{\gamma}\right\}$ must include gauge groups of arbitrarily large dimension. We divide the possibilities into two cases.

(1) The dimension of the simple factors in the groups $G_{\gamma}$ is bounded across all $\gamma$. In this case, the number of simple factors is unbounded over the family. 
(2) The dimension of at least one simple factor in $G_{\gamma}$ is unbounded. For example, the gauge group is of the form $G_{\gamma}=S U\left(N_{\gamma}\right) \times \tilde{G}_{\gamma}$, where $N_{\gamma} \rightarrow \infty$.

Case 1: In this case we can rule out infinite families with bounded values of $T$. The dimension of each factor $H_{i}$ is bounded, say by $\operatorname{dim} H_{i} \leq D$. Assume that we have an infinite sequence of models whose gauge groups have $N_{\gamma}$ factors, with $N_{\gamma}$ unbounded. Consider one model in this infinite sequence, with $N$ factors. We divide the factors $H_{i}$ into 3 classes, depending upon the sign of $b_{i}^{2}$ :

(1) Type Z: $b_{i}^{2}=0$

(2) Type N: $b_{i}^{2}<0$

(3) Type P: $b_{i}^{2}>0$

Since the dimension of each factor is bounded, the contribution to $H-V$ from $-V$ is bounded below by $-N D$. For fixed $T$ the total number of hypermultiplets is then bounded by

$$
H \leq 273-29 T+N D \equiv B \sim \mathcal{O}(N) .
$$

This means that the dimension of any irreducible component of $M$ is bounded by the same value $B$. The number of gauge group factors $\lambda$ under which any matter field can transform nontrivially is then bounded by $2^{\lambda} \leq B$, so $\lambda \leq \mathcal{O}(\ln N)$.

Now, consider the different types of factors. Denote the number of type $\mathrm{N}, \mathrm{Z}, \mathrm{P}$ factors by $N_{N, Z, P}$, where

$$
N=N_{N}+N_{Z}+N_{P} .
$$

We can write the $b_{i}$ 's in a (not necessarily integral) basis where the inner product matrix takes the form $\Omega=\operatorname{diag}(+1,-1,-1, \ldots)$ as

$$
b_{i}=\left(x_{i}, \vec{y}_{i}\right) \text {. }
$$

For any type $\mathrm{P}$ factor, $\left|x_{i}\right|>\left|\vec{y}_{i}\right|$, so $b_{i} \cdot b_{j}>0$ for any pair of type $\mathrm{P}$ factors. Thus, there are hypermultiplets charged under both gauge groups for every pair of type $\mathrm{P}$ factors. A hypermultiplet charged under $\lambda \geq 2$ gauge group factors appears in $\lambda(\lambda-1)$ (ordered) pairs $(i, j)$ with $b_{i} \cdot b_{j}>0$, and contributes at least $2^{\lambda}$ to the total number of hypermultiplets $H$. Each ordered pair under which this hypermultiplet is charged then contributes at least

$$
\frac{2^{\lambda}}{\lambda(\lambda-1)} \geq 1
$$

to the total number of hypermultiplets $H$. It follows that the $N_{P}\left(N_{P}-1\right)$ pairs under which at least one hypermultiplet is charged contribute at least $N_{P}\left(N_{P}-1\right)$ to $H$, so

$$
N_{P}\left(N_{P}-1\right) \leq B
$$


Thus,

$$
N_{P}<\sqrt{B}+1 \leq \mathcal{O}(\sqrt{N})
$$

which is much smaller than $N$ for large $N$. So most of the $b_{i}^{\prime} s$ associated with gauge group factors in any infinite family must be type $Z$ or type $N$.

Now consider type $\mathrm{N}$ factors. Any set of $r$ mutually orthogonal type $N$ vectors defines an $r$-dimensional negative-definite subspace of $\mathbb{R}^{1, T}$. This means, in particular, that we cannot have $T+1$ mutually orthogonal type $N$ vectors. If we have $N_{N}$ type $N$ vectors, we can define a graph whose nodes are the type $N$ vectors, where an edge connects every two nodes associated with perpendicular vectors. Turán's theorem [22] states that the maximum number of edges on any graph with $n$ vertices which does not contain a subset of $T+1$ completely connected vertices is

$$
(1-1 / T) n^{2} / 2
$$

where the total number of possible edges is $n(n-1) / 2$. Thus, applying this theorem to the graph described above on nodes associated with type $N$ vectors, the number of ordered pairs with charged hypermultiplets must be at least

$$
\frac{N_{N}^{2}}{T}-N_{N}
$$

It then follows that, assuming $T$ is fixed,

$$
N_{N} \leq \sqrt{T B}+T \sim \mathcal{O}(\sqrt{N}) .
$$

Finally, consider type $\mathrm{Z}$ factors. Vectors $b_{i}, b_{j}$ of the form (3.4) associated with two type $\mathrm{Z}$ factors each have $\left|x_{i}\right|=\left|\vec{y}_{i}\right|$ and have a positive inner product unless they are parallel, in which case $b_{i} \cdot b_{j}=0$. Denote by $\mu$ the size of the largest collection of parallel type $\mathrm{Z}$ vectors. Each type $\mathrm{Z}$ vector is perpendicular to fewer than $\mu$ other type $\mathrm{Z}$ vectors, so there are at least $N_{Z}\left(N_{Z}-\mu\right)$ ordered pairs of type $\mathrm{Z}$ factors under which there are charged hypers. We must then have

$$
N_{Z}\left(N_{Z}-\mu\right)=\left(N_{Z}-\mu\right)\left(N-N_{P}-N_{N}\right) \leq B .
$$

But from $(3.7,3.10)$ this means that $N_{Z}-\mu$ is of order at most $\mathcal{O}(1)$ (and is bounded by $D$ as $N \rightarrow \infty$ ), while $N_{Z}$ is of order $\mathcal{O}(N)$. Thus, all but a fraction of order $1 / N$ of the type $\mathrm{Z}$ factors have vectors in a common parallel direction. In [4], we carried out a case-by-case analysis demonstrating that all group + matter configurations which give type $\mathrm{Z}$ factors have a positive value for $H-V$. All of the $\mu$ factors associated with the largest collection of parallel type $\mathrm{Z}$ vectors thus contribute positively to $H-V$, counting matter charged under these group factors only once. The total contribution to $H-V$ is then bounded by

$$
H-V>\mu-\left[\left(N_{Z}-\mu\right)+N_{P}+N_{N}\right](D) \sim \mathcal{O}(N)
$$


TABLE 1. Allowed charged matter for an infinite family of models with gauge group $H(N)$. The last two columns give the values of $a \cdot b, b^{2}$ in the anomaly polynomial $I_{8}$.

\begin{tabular}{lccrr}
\hline Group & Matter content & $H-V$ & $a \cdot b$ & $b^{2}$ \\
\hline$S U(N)$ & $2 N \square$ & $N^{2}+1$ & 0 & -2 \\
& $(N+8) \square+1 \square$ & $\frac{1}{2} N^{2}+\frac{15}{2} N+1$ & 1 & -1 \\
& $(N-8) \square+1 \square$ & $\frac{1}{2} N^{2}-\frac{15}{2} N+1$ & -1 & -1 \\
& $16 \square+2 \square$ & $15 N+1$ & 2 & 0 \\
$S O(N)$ & $(N-8) \square$ & $\frac{1}{2} N^{2}-\frac{7}{2} N$ & -1 & -1 \\
$S p(N / 2)$ & $(N+8) \square$ & $\frac{1}{2} N^{2}+\frac{7}{2} N$ & 1 & -1 \\
& $16 \square+1 \square$ & $15 N-1$ & 2 & 0 \\
\hline & & & &
\end{tabular}

which exceeds the bound $H-V \leq 273-29 T$ for sufficiently large $N$. Thus, we have ruled out case 1 by contradiction for any fixed $T>0$, and in consequence ruled out any infinite family of the type of case 1 with bounded values of $T$.

Case 2: We now consider the possibility of infinite families with factors of unbounded size. We assume that we have an infinite family of models with gauge groups of the form $G_{\gamma}=H_{\gamma} \times \tilde{G}_{\gamma}$ where $\operatorname{dim} H_{\gamma} \rightarrow \infty$. For $S U(N)$ the $F^{4}$ anomaly cancellation condition

$$
B_{\text {Adj }}=2 N=\sum_{R} x_{R} B_{R}
$$

can only be satisfied at large $N$ when the number of multiplets $x_{R}$ vanishes in all representations other than the fundamental, adjoint, and two-index antisymmetric and symmetric representations (we consider representations and their conjugates to be equivalent for the purposes of this analysis). This follows from the fact that for all other representations, $B_{R}$ grows faster than $N$. For the representations just listed, indexed in that order, (3.13) becomes

$$
2 N=x_{1}+2 N x_{2}+(N-8) x_{3}+(N+8) x_{4} .
$$

The solutions to this equation at large $N$, along with the corresponding solutions for the other classical groups $S O(N), S p(N)$ are easy to tabulate, and are listed in Table 1 . We discard solutions $\left(x_{1}, x_{2}, x_{3}, x_{4}\right)=(0,1,0,0)$ and $(0,0,1,1)$, where $a \cdot b_{i}=b_{i}^{2}=0$ since for $T<9$ these relations combined with $a^{2}>0$ imply that $b_{i}=0$ and therefore that the kinetic term for the gauge field is identically zero. The contribution to $H-V$ from each of the group and matter combinations in Table 1 diverges as $N \rightarrow \infty$. This cannot be cancelled by contributions to $-V$ from an infinite number of factors, for the same reasons which rule out case 1 . Thus, any infinite family must have an infinite sub-family, with gauge group of the form $\hat{H}(M) \times H(N) \times \tilde{G}_{M, N}$, 
with both $M, N \rightarrow \infty$. For any factors $H_{i}, H_{j}$ with $a \cdot b_{i}, a \cdot b_{j} \neq 0$, in a (non-integral) basis where $\Omega=\operatorname{diag}(+1,-1,-1, \ldots)$, and $a=\left(\sqrt{a^{2}}, 0,0, \ldots\right)$ writing

$$
b_{i}=\left(x_{i}, \vec{y}_{i}\right)
$$

with $x_{i}=a \cdot b_{i} / \sqrt{a^{2}}$ we have

$$
x_{i} x_{j}=\left(a \cdot b_{i}\right)\left(a \cdot b_{j}\right) / a^{2} \geq b_{i} \cdot b_{j}=\sum_{R, S} x_{R S} A_{R} A_{S} .
$$

Since $x_{i} x_{j}$ is constant for any infinite family of pairs $\hat{H}(M), H(N)$, while $A_{R}$ grows for all representations besides the fundamental, the only possible fields charged under more than one of the infinite factors in Table 1 are bifundamentals.

We now consider all possible infinite families built from products of groups and representations in Table 1 which have bifundamental fields and bounded $H-V$. There are 5 such combinations with two factors. These combinations were enumerated in [4], and are listed in Table 4 in that paper. These combinations include two infinite families shown to satisfy anomaly factorization by Schwarz [21], as well as three other similar families. For example, the simplest such family consists of an infinite sequence of models with gauge group $S U(N) \times S U(N)$ and two matter fields transforming in the bifundamental $(N, N)$ representation. This model has $H=2 N^{2}, V=$ $2\left(N^{2}-1\right)$ so $H-V=2$ for each model in the infinite family. In addition to the infinite families built from products of two factors with unbounded dimension, when $T>1$ there are several infinite families arising from products of three factors with unbounded dimension. For example, there is such a family of models with gauge group $G=S U(N-8) \times S U(N) \times S U(N+8)$. The other possibilities are described in $[6]$. For $T<9$, the models in all of these infinite families with unbounded factors are unacceptable because the gauge kinetic term for one of the factors is always unphysical. This can be shown as follows: For each two-factor infinite family we have two vectors $b_{1}, b_{2}$ which satisfy $a \cdot\left(b_{1}+b_{2}\right)=0$ and $\left(b_{1}+b_{2}\right)^{2}=0$. But when $a^{2}>0$ these conditions imply $b_{1}+b_{2}=0$, so that $j \cdot b_{1}$ and $j \cdot b_{2}$ cannot both be positive. For example, for the theory found by Schwarz with gauge group $S U(N) \times S U(N)$ with two bifundamental fields, we have $a \cdot b_{1}=a \cdot b_{2}=0, b_{1}^{2}=b_{2}^{2}=-2, b_{1} \cdot b_{2}=2$, from which it follows that $b_{1}=-b_{2}$. Essentially the same argument works for each three-factor infinite family, where $a \cdot\left(b_{1}+b_{2}+b_{3}\right)=0$. This method of proof breaks down when $T>8$, where $a^{2} \leq 0$, since then $a \cdot b=b^{2}=0$ is not sufficient to prove $b=0$. In the following section we give an example of an infinite family of models with no clear inconsistency at $T=9$.

This proves case 2 of the analysis. So we have proven that for $T<9$ there are a finite number of distinct gauge groups and matter content which satisfy anomaly cancellation with physical kinetic terms for all gauge field factors. We have ruled out infinite families with unbounded numbers of 
gauge group factors at any finite $T$, though infinite families with unbounded numbers of factors satisfying the anomaly and group theory sign constraints can exist when $T$ is unbounded, as discussed in [6]. We have not ruled out infinite families with a finite number of gauge group factors which become unbounded at finite $T>8$. Indeed, we give an explicit construction of such a family in Section 5.

Theorem (3.1) strongly constrains the range of possible supergravity theories, at least when $T<9$. Furthermore, the analysis in the proof of the theorem can be used to give explicit algorithms for systematically enumerating all the possible gauge groups and matter content which can appear in acceptable theories, as discussed in $[\mathbf{4}, \mathbf{5}]$.

\section{6D supergravities from F-theory}

4.1. F-theory models from elliptically fibered Calabi-Yau threefolds. There are many ways in which string theory can be used to construct supergravity theories in various dimensions. In general, such constructions involve starting with a 10-dimensional string theory, and "compactifying" the theory by wrapping $10-D$ dimensions of the space-time on a compact $(10-D)$-dimensional manifold to give a theory in $D$ dimensions which behaves as a supergravity theory at low energies. At this point in time, we do not have any fundamental "background-independent" definition of string theory, in which all the different compactifications of the theory arise on equal footing. Rather, string theory consists of an assemblage of tools, including low-energy supergravity, perturbative string theory, and nonperturbative structures such as D-branes and duality symmetries, which give an apparently consistent way of constructing and relating the various approaches to string compactification.

One of the most general approaches to string compactification is "F-theory" $[\mathbf{8}, \mathbf{9}]$. Technically, F-theory describes certain limits of string compactifications in which certain information (such as Kähler moduli) is lost. Mathematically, an F-theory compactification to $D$ dimensions is characterized by an elliptically fibered Calabi-Yau manifold of dimension $12-D$. The data needed to define an F-theory compactification to six dimensions consist of a complex surface $B$ which acts as the base of the fibration, and an elliptic fibration with section over $B$. The elliptic fibration may have singularities, as long as such singularities can be resolved to give a total space which is Calabi-Yau. The structure of such an elliptic fibration is generally described by a Weierstrass model

$$
y^{2}=x^{3}+f(u, v) x z^{4}+g(u, v) z^{6}
$$

where $u, v$ are coordinates on the base $B$. The functions $f, g$ are sections of the line bundles $-4 K$ and $-6 K$ respectively, where $K$ is the canonical bundle of $B$. 
The gauge group and matter structure of the low-energy theory associated with an F-theory compactification on an elliptically fibered Calabi-Yau threefold are determined by the singularity structure of the fibration. Simple nonabelian gauge group factors $H_{i}$ in the associated 6D theory are associated with codimension one loci in the base where the fiber degenerates. Such codimension one singularities were classified by Kodaira [23]. These singularities can be associated with A-D-E Dynkin diagrams and give rise to the corresponding simply-laced gauge group factors. Nontrivial monodromies about codimension 2 singularities expand the possible gauge group factors to include the non-simply laced groups, $S p(N), F_{4}$ and $G_{2}[\mathbf{2 5}]$. Given a Weierstrass form for the elliptic fibration, the singularity types can be determined in terms of the orders of vanishing of $f, g$, and the discriminant locus

$$
\Delta=4 f^{3}+27 g^{2},
$$

which is a section of $-12 \mathrm{~K}$. The resulting gauge group can be then determined via Tate's algorithm [24]. For example, a singularity of Kodaira type $I_{n}$, corresponding to the Dynkin diagram $A_{n-1}$, arises on a codimension one singularity locus where neither $f$ nor $g$ vanishes, but $\Delta$ vanishes to order $n$. Such a singularity gives a gauge group factor $S U(n)$ when the base of the fibration is (complex) one-dimensional, and $S U(n)$ or $S p(n / 2)$ in the low-energy $6 \mathrm{D}$ theory when the base is 2 dimensional. A complete list of the possible singularity types and associated orders of vanishing of $f, g, \Delta$ can be found in, for example, $[\mathbf{9}, \mathbf{2 5}]$. Each nonabelian gauge group factor $H_{i}$ is associated with a singularity locus on an effective irreducible divisor $\xi_{i} \in H_{2}(B ; \mathbb{Z})$.

Matter fields (hypermultiplets) in the low-energy 6D theory are associated with codimension two singularities in the elliptic fibration. Generally such singularities arise at intersections of divisors associated with codimension one singularities. For example, an $A_{n-1}$ singularity intersecting an $A_{m-1}$ singularity gives rise to a codimension two $A_{n+m-1}$ singularity, where $\Delta$ vanishes to order $n+m$. The resulting matter fields transform in the fundamental of the associated $S U(n)$ gauge group factor and the (anti)-fundamental of the associated $S U(m)$ gauge group factor. (This can be thought of as splitting the adjoint representation of $S U(n+m)$ as a representation of $S U(n) \times S U(m))$. The singularities associated with possible matter representations which can arise in this fashion have not been fully classified, but an analysis of many such representations appears in $[\mathbf{2 7}, \mathbf{2 8}]$.

As discussed in [9], it was shown by Kodaira for a base of complex dimension 1, and subsequently generalized to higher dimension by other authors, that the total space of the elliptic fibration can be resolved to a Calabi-Yau manifold when $-12 K=\Delta$, where $\Delta$ is the total divisor class of the singular (discriminant) locus. The component of the discriminant locus for each nonabelian factor is given by an effective irreducible divisor $\xi_{i}$ with a multiplicity $\nu_{i}$. For example, for an $A_{n-1}$ singularity, $\nu_{i}=n$. The residual singularity locus, which is not associated with nonabelian gauge 
group structure, is associated with another effective divisor $Y$, so that the Kodaira relation is

$$
-12 K=\Delta=\sum_{i} \nu_{i} \xi_{i}+Y
$$

In this fashion, a wide range of $6 \mathrm{D}$ supergravity theories can be constructed from particular choices of elliptically fibered Calabi-Yau threefolds. In all such situations, the resulting gauge group and hypermultiplet matter content automatically satisfy the anomaly cancellation conditions $[\mathbf{2 6}, \mathbf{2 7}]$. We now discuss the connection between the data of the low-energy theory and that of the F-theory compactification.

4.2. Mapping 6D supergravities to F-theory. To identify the set of low-energy 6D supergravity models which are compatible with F-theory, we would like to have a systematic approach to constructing an F-theory model which realizes a particular low-energy gauge group $G$ and matter representation $M$. In fact, as shown in $[\mathbf{2 6}, \mathbf{2 7}]$, the anomaly cancellation conditions give a close correspondence between the data of a geometric F-theory construction and the corresponding $6 \mathrm{D}$ supergravity theory. This correspondence characterizes a map from low-energy data to topological F-theory data $[\mathbf{5}, \mathbf{6}]$; identifying those cases where such a map cannot be consistently defined allows us to determine which $G, M$ cannot be found through any F-theory construction.

The connection between the $6 \mathrm{D}$ supergravity theory and F-theory data is given by the following correspondences $[\mathbf{5}, \mathbf{6 , 2 6 , 2 7}]$ : the number of tensor multiplets $T$ in the $6 \mathrm{D}$ theory is equal to $h^{1,1}-1$ of the base $B$ of the F-theory elliptic fibration. The lattice $\Lambda$ determined by the anomaly cancellation conditions through $(2.10-2.12)$ can be embedded into the integral second homology of $B$

$$
\Lambda \hookrightarrow H_{2}(B ; \mathbb{Z})
$$

in such a way that

$$
\begin{aligned}
a & \rightarrow K \\
b_{i} & \rightarrow \xi_{i}
\end{aligned}
$$

Thus, for example, we have

$$
a \cdot a=9-T=10-h^{1,1}=K^{2} .
$$

For an elliptic fibration which resolves to a smooth Calabi-Yau threefold, the possible smooth bases $B$ consist of $\mathbb{P}^{2}$ and the Hirzebruch surfaces $\mathbb{F}_{m}$ $(m \leq 12)$ and blow-ups of these bases, as well as the somewhat trivial cases of $\mathrm{K} 3$ and the Enriques surface [9]. For example, at $T=1$, the set of possible F-theory bases are $\mathbb{F}_{m}, m \leq 12$. In this case, a basis for $H_{2}(B ; \mathbb{Z})$ is given by 
$D_{v}, D_{s}$ where $D_{v}^{2}=-m, D_{v} \cdot D_{s}=1, D_{s}^{2}=0$. As shown in [5], in this case, the vector $b$ associated with a given gauge group factor maps to the divisor

$$
b \rightarrow \xi=\frac{\alpha}{2}\left(D_{v}+\frac{m}{2} D_{s}\right)+\frac{\tilde{\alpha}}{2} D_{s}
$$

where $\alpha, \tilde{\alpha}$ satisfy $-a \cdot b=\alpha+\tilde{\alpha}, b^{2}=\alpha \tilde{\alpha} / 2$.

In the following section we give some explicit examples of $6 \mathrm{D}$ supergravity theories and the corresponding F-theory constructions. In some cases, there is no map of the form (4.4) where $a, b_{i}$ map to effective divisors in any $B$ with the correct properties for an F-theory compactification. For these models, which are apparently consistent from the low-energy.of view and yet have no F-theory construction, we can identify which aspect of the F-theory structure breaks down. We include some examples of this type in the following section. In some other cases, there are multiple possible F-theory realizations of the specified $6 \mathrm{D}$ gauge group $G$ and matter representation $M$. Thus, the correspondence does not give a uniquely defined map from low-energy data to F-theory. It does, however, give us a framework for analyzing which low-energy theories admit an F-theory realization. (Note that in those cases where the gauge group and matter content are not sufficient to uniquely determine the F-theory construction, further information about the low-energy theory may single out a particular F-theory model.)

\section{Examples}

In this section we consider some examples of possible gauge groups and matter representations for $6 \mathrm{D}$ supergravities. We begin with two examples of theories which seem to admit consistent embeddings in F-theory, and then consider two specific examples and one infinite family of theories which cannot be realized using standard F-theory techniques (or any other known string construction).

ExAmPle 1. $T=0, G=S U(N), M=3 \times \square+(24-N) \times \square$

A simple class of examples involve theories with no tensor multiplets $(T=0)$. In this case, $a^{2}=9$, and the space $\mathbb{R}^{1, T}=\mathbb{R}^{1}$ is just one-dimensional Euclidean space, so $-a=(3)$ in an integral basis. Any $S U(N)$ factor is associated with a vector $b=(k)$. For simplicity, we assume that the only matter consists of $F$ hypermultiplets transforming in the fundamental representation and $A$ hypermultiplets in the two-index antisymmetric representation $(\square)$. We can use the group theory coefficients

$$
\begin{array}{lll}
A_{f}=1, & B_{f}=1, & C_{f}=0 \\
A_{a}=N-2, & B_{a}=N-8, & C_{a}=3 \\
A_{\text {Adj }}=2 N, & B_{\text {Adj }}=2 N, & C_{\text {Adj }}=6
\end{array}
$$


for $S U(N)$ to write the anomaly cancellation equations:

$$
\begin{aligned}
0 & =2 N-F-A(N-8) \\
-a \cdot b=3 k & =-(2 N-F-A(N-2)) / 6=A \\
b^{2}=k^{2} & =(-6+3 A) / 3=A-2
\end{aligned}
$$

For $k=1$, we have solutions for $A=3$, with $F=24-N$ for $N \leq 24$. This gives a set of models with simple gauge group $G=S U(N)$ which satisfies all anomaly cancellation conditions. The gauge kinetic term condition is satisfied for any positive $j=\left(j_{0}\right)$.

For the corresponding F-theory construction, we have $\mathbb{P}^{2}$ as the only possible base with $h^{1,1}=T+1=1$. The second homology of $\mathbb{P}^{2}$ is generated by the cycle $H$, corresponding to the hyperplane divisor, satisfying $H \cdot H=1$. The map from $\Lambda \hookrightarrow H_{2}(B ; \mathbb{Z})$ is then given by the trivial map (1) $\rightarrow H$, so that

$$
\begin{aligned}
-a & =(3) \rightarrow 3 H=-K \\
b & =(1) \rightarrow H .
\end{aligned}
$$

This gives the topological data associated with the corresponding F-theory construction. To verify that there exists an F-theory model from an elliptic fibration associated with this data one must construct a Weierstrass model with singularity type $A_{N-1}$ on the divisor $H$. We discuss explicit construction of Weierstrass models in the following section. One can similarly construct a variety of anomaly-free models with gauge group of the form $G=\prod_{i} S U\left(N_{i}\right)$ and matter in fundamental, antisymmetric, and bifundamental representations. We explicitly constructed all such models for $T=1$ in $[\mathbf{5}]$ and found that all appear to correspond to acceptable topological data for F-theory constructions.

ExAmple 2. $T=1, G=E_{8} \times E_{7}$

This gauge group has two simple factors, and thus two vectors $b_{8}, b_{7}$ in $\mathbb{R}^{1,1}$. For $E_{8}$ and $E_{7}$ we have the normalization factors $\lambda_{8}=60, \lambda_{7}=12$. Neither $E_{8}$ or $E_{7}$ has a fourth order invariant, so (2.6) is automatic. Assume we have $F$ matter fields in the fundamental representation of $E_{7}$, with no matter transforming under the $E_{8}$ (the fundamental representation for $E_{8}$ is equivalent to the adjoint). The $E_{8}$ anomaly equations state that

$$
-a \cdot b_{8}=-10, \quad b_{8}^{2}=-12
$$

where $a^{2}=8$. In a basis where

$$
\Omega_{\alpha \beta}=\left(\begin{array}{ll}
0 & 1 \\
1 & 0
\end{array}\right)
$$

we can choose $-a=(2,2)$, and $b_{8}=(1,-6)$. The anomaly equations for $E_{7}$, including the $\operatorname{tr} F_{7}^{2} \operatorname{tr} F_{8}^{2}$ condition, state that

$$
-a \cdot b_{7}=2 F-6, \quad b_{7}^{2}=2 F-8, \quad b_{7} \cdot b_{8}=0 .
$$


These equations have the unique solution $b_{7}=(1,6)$, realized when $F=10$. Thus, the anomaly cancellation conditions for this class of theory are only realized for this value of $F$. In this case we can read the corresponding F-theory model directly from (4.8):

$$
\begin{aligned}
& b_{8}=\frac{1}{2}\left(\alpha_{8}, \tilde{\alpha}_{8}\right) \rightarrow \xi_{8}=D_{v}+(m / 2-6) D_{s} \\
& b_{7}=\frac{1}{2}\left(\alpha_{7}, \tilde{\alpha}_{7}\right) \rightarrow \xi_{7}=D_{v}+(m / 2+6) D_{s}
\end{aligned}
$$

which gives effective irreducible divisors precisely when $m=12$. In this case we have an acceptable F-theory construction on the base manifold $\mathbb{F}_{12}[\mathbf{9}]$, with $\xi_{8}=D_{v}, \xi_{7}=D_{u}=D_{v}+12 D_{s}$. This model was identified in the corresponding heterotic string formulation by Seiberg and Witten in [29].

We now present several models which seem consistent from the point of view of anomalies and gauge kinetic terms, but which have no known string theory realization.

ExAmple 3. $T=1, G=S U(4), M=\operatorname{Adj}+10 \times \square+40 \times \square$

For this model the anomaly cancellation conditions give

$$
\Lambda=\left(\begin{array}{cc}
a^{2} & -a \cdot b \\
-a \cdot b & b^{2}
\end{array}\right)=\left(\begin{array}{cc}
8 & 10 \\
10 & 10
\end{array}\right)
$$

This lattice cannot be embedded in any unimodular lattice [6]. Since the intersection form on $H_{2}(B ; \mathbb{Z})$ must give a unimodular lattice by Poincaré duality, this model cannot be embedded in F-theory.

Example 4. $T=1, G=S U(8), M=\square$

Using the coefficients $A=10, B=16, C=3$ for the symmetric representation, we find that the anomaly conditions give

$$
\Lambda=\left(\begin{array}{cc}
a^{2} & -a \cdot b \\
-a \cdot b & b^{2}
\end{array}\right)=\left(\begin{array}{cc}
8 & -1 \\
-1 & -1
\end{array}\right)
$$

While this lattice can be embedded in $H_{2}(B ; \mathbb{Z})$ for some F-theory bases, the resulting divisor $b$ is not an effective irreducible divisor. For example, we could choose $-a \rightarrow-K=2 D_{v}+3 D_{s}$ on $\mathbb{F}_{1}$ giving $b \rightarrow-D_{v}$. But since the divisors $\xi_{i}$ carrying the singularity locus on the F-theory base must be irreducible effective divisors, this will not work for constructing an F-theory realization of this model using existing methods.

ExAmple 5. $T=9, G=S U(N) \times S U(N), M=2 \times(\square, \square)$

For this gauge group and matter representations, at $T=9$ we need vectors $-a, b_{1}, b_{2}$ with inner product matrix

$$
\Lambda=\left(\begin{array}{ccc}
0 & 0 & 0 \\
0 & -2 & 2 \\
0 & 2 & -2
\end{array}\right)
$$


In a basis with $\Omega=\operatorname{diag}(+1,-1,-1, \ldots)$, this can be realized through the vectors

$$
\begin{aligned}
-a & =(3,-1,-1,-1,-1,-1,-1,-1,-1,-1) \\
b_{1} & =(1,-1,-1,-1,0,0,0,0,0,0) \\
b_{2} & =(2,0,0,0,-1,-1,-1,-1,-1,-1)
\end{aligned}
$$

This satisfies the correct gauge kinetic term sign conditions $j \cdot b_{i}>0$ for $j=(1,0,0, \ldots)$. Thus, this infinite family of models cannot be ruled out by the low-energy constraints we have imposed here.

These models are not valid in F-theory, however, when $N>12$, since in that case

$$
Y=-12 K-N\left(\xi_{1}+\xi_{2}\right)=(N-12) K
$$

which cannot be effective for $N>12$ since $-K$ is effective.

\section{String universality?}

The results presented so far demonstrate that, although anomaly cancellation and other known constraints provide substantial limits on the range of gauge groups and matter representations that can be realized in supersymmetric six-dimensional quantum theories of gravity, not all models which satisfy these constraints can at this time be realized in string theory. Some of the examples presented in the previous section seem like counterexamples to the string universality conjecture stated in the introduction. At this time, however, our understanding of the situation is still incomplete. We do not have a complete definition of string theory that allows us to determine with certainty which low-energy models can or cannot be realized within string theory. It is also quite possible that string theory imposes additional constraints, like the sign condition on the gauge kinetic terms, which may be seen as quantum consistency conditions given the data of the low-energy theory. To prove or disprove the conjecture of string universality in six dimensions, it seems that further progress is needed in understanding both string theory and low-energy supergravity. There are also some purely mathematical questions whose solutions may contribute significantly to making progress in this direction. In this concluding section, we summarize some of the most relevant open questions, both mathematical and physical, in this regard. Most of these questions are discussed in further detail in [6].

6.1. Mathematics questions. There are a number of concrete mathematical questions related to F-theory whose solutions would help clarify the precise set of theories which can be realized in string theory via F-theory. We briefly summarize here a few of these questions that are most closely related to the discussion in this paper. 


\section{Weierstrass models}

We have argued that the correspondence between the low-energy data associated with the anomaly cancellation conditions and the mathematical structure of F-theory leads to a map (4.4) from the anomaly lattice $\Lambda$ to the second homology lattice of the F-theory base $B$. In many cases this map associates with each nonabelian factor in the gauge group an effective divisor on $B$. In order to show that the model can be realized in F-theory, however, it is necessary to construct an explicit Weierstrass model with the desired singularity locus associated with these divisors. In many cases it seems that this can be done. For a class of models with $T=1$ and gauge group $S U(N)$, we showed in [5] that there is a precise correspondence between the number of degrees of freedom needed to fix the desired singularity structures on the proper divisor locus and the number of degrees of freedom encoded in the scalar matter fields through $H-V$. It may be possible to give a mathematical proof that the existence of a Weierstrass model follows given some necessary topological conditions on the divisor locus. Such a proof would be a useful step forward in identifying a large class of the anomaly-free models which have F-theory realizations.

\section{Classifying matter singularities}

As mentioned above, while codimension one singularities in elliptic fibrations are well-understood, codimension two singularities arising at the intersection of codimension one singularities are not completely understood. Such singularities give rise in F-theory compactifications to a variety of different representation structures for matter fields. In studying the set of anomaly-free supergravity theories in six dimensions, we have encountered some novel matter representations which may or may not be associated with valid $\mathrm{F}$-theory compactifications. For example, in [5], we found some models with an $S U(N)$ gauge group and matter in a four-index antisymmetric representation. While some F-theory constructions are known which give rise to three-index antisymmetric representations of $S U(N)$ [28] there is as yet no understanding of a singularity structure which would give a four-index antisymmetric representation. A complete classification of codimension two singularities in elliptic fibrations of Calabi-Yau manifolds would be of great assistance in systematically understanding the set of allowed F-theory compactifications.

\section{Classification of elliptically fibered Calabi-Yau manifolds}

It has been shown by Gross [30] that the number of distinct topological types of elliptically fibered Calabi-Yau threefolds is finite up to birational equivalence. Finiteness of the set of topologically distinct elliptically-fibered Calabi-Yau threefolds is shown in [6] using minimal surface theory and the fact that the Weierstrass form for an elliptic fibration over a fixed base has a finite number of possible distinct singularity structures. These arguments, however, do not give a clear picture of how such compactifications 
can be systematically classified. A complete mathematical classification of elliptically fibered Calabi-Yau threefolds would be helpful in understanding the range of F-theory compactifications. The analogue of this question for four dimensions, while probably much more difficult, would be of even greater interest, since at this time we have very little handle on the scope of the space of four dimensional supergravity theories which can be realized through F-theory compactifications on Calabi-Yau fourfolds.

6.2. Physics questions. Some of the most difficult questions which need to be answered to prove or disprove the conjecture of string universality in six dimensions are essentially physical in nature. The key questions amount to

A) Are there as-yet unknown string compactifications that extend the range of $6 \mathrm{D}$ theories beyond those attainable using known F-theory constructions?

B) Can we identify new quantum consistency constraints on low-energy theories that constrain the space of allowed models to match more closely the set which can be realized through string compactifications?

While A) is certainly an important question, it seems that the most likely opportunity for narrowing the gap between the space of allowed theories and the space of string constructions is by identifying new constraints on low-energy theories. If string universality is even approximately correct, it is probably necessary to find a low-energy quantum consistency condition which rules out the infinite families of theories compatible with anomaly cancellation but which cannot be realized in F-theory. One place to look for such constraints is in the set of conditions imposed by F-theory. Considering the examples 3-5 in the previous section which cannot be realized in F-theory, we can identify some possible constraints that may be needed in addition to anomaly cancellation and the gauge kinetic term sign constraint. It seems plausible that some of these constraints may in fact be needed for quantum consistency of any low-energy theory. We briefly mention here a few of these constraints, and make a few comments on how they may be realized.

\section{Unimodular lattice embedding}

As we see from example 3, a necessary condition for a given low-energy theory to be realizable through F-theory is that the lattice $\Lambda$ associated with the anomaly cancellation constraints must be embeddable in a unimodular lattice. Associated with any of the supersymmetric six dimensional gravity theories we are considering here, there is a lattice of dyonic strings charged under the anti-symmetric tensor fields of the theory, carrying a natural inner product of signature $(1, T)$. Some aspects of this lattice are discussed in [6]. We do not know any reason why this lattice must be unimodular for quantum consistency, but it is possible that this may be necessary for unitary of the theory, or for some other consistency reason. Note that an 
analogous unimodularity constraint follows from modular invariance for the heterotic fundamental string construction. Demonstrating that the string dyonic lattice in a general $6 \mathrm{D}$ theory needs to be unimodular for a quantum theory to exist would help in ruling out some of the apparently consistent theories which we do not know how to realize in F-theory.

\section{Kodaira constraint and effective divisors}

As we noted in examples 4 and 5, some apparently consistent theories cannot be realized in F-theory because the topological data for the F-theory model realized through (4.4) leads to certain divisors in F-theory not satisfying an effectiveness condition which is needed for the F-theory construction. In particular, the divisors $-K, \xi_{i}$ associated with the vectors $-a, b_{i}$ must all be effective (inside the Mori cone), as must the residual divisor locus $Y$ associated with the vector $12 a-\sum_{i} \nu_{i} b_{i}$. This condition has no obvious counterpart in the low-energy theory. It seems plausible, however, that some of these conditions may be associated with sign constraints in the low-energy theory analogous to the sign constraint on the gauge kinetic term. For example, a term proportional to $a \cdot j \operatorname{tr} R^{2}$ must appear in the action of the theory by supersymmetry. Arguments analogous to those in [31] may suggest that this term must have a particular sign for consistency with causality in field theory. It is less clear how the condition on the residual divisor locus can be seen from the low-energy theory, but it seems possible that such a condition would arise from consistency with supersymmetry, since this is the ultimate origin of the Kodaira/Calabi-Yau condition in F-theory.

To conclude, we have not yet proven or disproven the conjecture of string universality in six dimensions. Simply pursuing this conjecture, however, has given new insights into the global structure of the space of $6 \mathrm{D}$ supergravity theories and string compactifications, and has suggested some intriguing avenues for future progress, both in physics, and in mathematics.

\section{References}

[1] M. R. Douglas and S. Kachru, "Flux compactification," Rev. Mod. Phys. 79, 733 (2007) arXiv:hep-th/0610102.

[2] C. Vafa, "The string landscape and the swampland," arXiv:hep-th/0509212.

[3] V. Kumar and W. Taylor, "String Universality in Six Dimensions," arXiv:0906.0987 [hep-th].

[4] V. Kumar and W. Taylor, "A bound on $6 \mathrm{D} \mathcal{N}=1$ supergravities," JHEP 0912, 050 (2009) arXiv:0910.1586 [hep-th].

[5] D. R. Morrison, V. Kumar and W. Taylor, "Mapping $6 \mathrm{D} \mathcal{N}=1$ supergravity to F-theory," arXiv:0911.3393 [hep-th].

[6] D. R. Morrison, V. Kumar and W. Taylor, "Global aspects of the space of $6 \mathrm{D} \mathcal{N}=1$ supergravities," arXiv:1008.1062 [hep-th].

77] A. Adams, O. DeWolfe, and W. Taylor, "String universality in ten dimensions," arXiv:1006.1352 [hep-th].

[8] C. Vafa, "Evidence for F-Theory," Nucl. Phys. B 469, 403 (1996) arXiv:hepth/9602022.

[9] D. R. Morrison and C. Vafa, "Compactifications of F-Theory on Calabi-Yau Threefolds - I," Nucl. Phys. B 473, 74 (1996) arXiv:hep-th/9602114. 
D. R. Morrison and C. Vafa, "Compactifications of F-Theory on Calabi-Yau Threefolds - II," Nucl. Phys. B 476, 437 (1996) arXiv:hep-th/9603161.

[10] H. Nishino and E. Sezgin, "Matter And Gauge Couplings Of N=2 Supergravity In Six-Dimensions," Phys. Lett. B 144, 187 (1984).

H. Nishino and E. Sezgin, "The Complete $N=2, D=6$ Supergravity With Matter And Yang-Mills Couplings," Nucl. Phys. B 278, 353 (1986).

[11] L. J. Romans, "Self-duality for interacting fields: covariant field equations for six dimensional chiral supergravities," Nucl. Phys. B 276, 71 (1986).

[12] M. B. Green, J. H. Schwarz and P. C. West, "Anomaly Free Chiral Theories In SixDimensions," Nucl. Phys. B 254, 327 (1985).

[13] K. Fujikawa, Phys. Rev. Lett. 42, 1195 (1979); Phys. Rev. D21, 2848 (1980).

[14] L. Alvarez-Gaume and E. Witten, "Gravitational Anomalies," Nucl. Phys. B 234, 269 (1984).

[15] M. F. Atiyah and I. M. Singer, "Dirac operators coupled to vector potentials," Proc. Natl. Acad. Sci. 81 2597-600 (1984).

[16] L. Alvarez-Gaumé and P. Ginsparg, Ann. Phys. 161423 (1985)

[17] M. B. Green and J. H. Schwarz, "Anomaly Cancellation In Supersymmetric D=10 Gauge Theory And Superstring Theory," Phys. Lett. B 149, 117 (1984).

[18] M. B. Green, J. H. Schwarz and P. C. West, "Anomaly Free Chiral Theories In SixDimensions," Nucl. Phys. B 254, 327 (1985).

[19] A. Sagnotti, "A Note on the Green-Schwarz mechanism in open string theories," Phys. Lett. B 294, 196 (1992) arXiv:hep-th/9210127.

[20] J. Erler, "Anomaly Cancellation In Six-Dimensions," J. Math. Phys. 35, 1819 (1994) arXiv:hep-th/9304104.

[21] J. H. Schwarz, "Anomaly-Free Supersymmetric Models in Six Dimensions," Phys. Lett. B 371, 223 (1996) arXiv:hep-th/9512053.

[22] P. Turán . "On an extremal problem in graph theory," Matematikai és Fizikai Lapok 48: 436-452 (1941).

[23] K. Kodaira, Ann. Math. 77563 (1963); Ann. Math. 781 (1963).

[24] J. Tate, in: Modular functions of one variable IV, Lecture Notes in Math, vol. 476, Springer-Verlag, Berlin (1975).

[25] M. Bershadsky, K. A. Intriligator, S. Kachru, D. R. Morrison, V. Sadov and C. Vafa, "Geometric singularities and enhanced gauge symmetries," Nucl. Phys. B 481, 215 (1996) arXiv:hep-th/9605200.

[26] V. Sadov, "Generalized Green-Schwarz mechanism in F theory," Phys. Lett. B 388, 45 (1996) arXiv:hep-th/9606008.

[27] A. Grassi, D. R. Morrison, "Group representations and the Euler characteristic of elliptically fibered Calabi-Yau threefolds", J. Algebraic Geom. 12, 321-356 (2003) arXiv:math/0005196.

[28] A. Grassi, D. R. Morrison, "Anomalies and the Euler characteristic of elliptically fibered Calabi-Yau threefolds," to appear.

[29] N. Seiberg and E. Witten, "Comments on String Dynamics in Six Dimensions," Nucl. Phys. B 471, 121 (1996) arXiv:hep-th/9603003.

[30] M. Gross, "A finiteness theorem for elliptic Calabi-Yau threefolds," Duke Math. J. 74, 2, 271-299 (1994) arXiv:alg-geom/9305002.

[31] A. Adams, N. Arkani-Hamed, S. Dubovsky, A. Nicolis and R. Rattazzi, "Causality, analyticity and an IR obstruction to UV completion," JHEP 0610, 014 (2006) arXiv:hep-th/0602178.

Center for Theoretical Physics, Department of Physics, Massachusetts Institute of Technology; 77 Massachusetts Avenue, Cambridge, MA 02139, USA

E-mail address: wati@mit.edu 